\title{
Introduction to research topic - binocular rivalry: a gateway to studying consciousness
}

\author{
Alexander Maier ${ }^{1}$, Theofanis I. Panagiotaropoulos ${ }^{2}$, Naotsugu Tsuchiya ${ }^{3,4,5,6,7}$ and Georgios A. Keliris ${ }^{2,8 *}$ \\ 1 Department of Psychology, Vanderbilt University, Nashville, TN, USA \\ 2 Department of Physiology of Cognitive Processes, Max Planck Institute for Biological Cybernetics, Tübingen, Germany \\ ${ }^{3}$ School of Psychology and Psychiatry, Monash University, Clayton, Melbourne, VIC, Australia \\ ${ }^{4}$ Decoding and Controlling Brain Information, Japan Science and Technology Agency, Chiyoda-ku, Tokyo, Japan \\ ${ }^{5}$ Computational Neuroscience Laboratories, Advanced Telecommunications Research Institute International, Seika-shi, Kyoto, Japan \\ ${ }^{6}$ Brain Science Institute, RIKEN, Wako-shi, Saitama, Japan \\ 7 Division of Biology, California Institute of Technology, Pasadena, CA, USA \\ 8 Bernstein Center for Computational Neuroscience, Tübingen, Germany \\ *Correspondence: georgios.keliris@tuebingen.mpg.de \\ Edited by: \\ Hauke R. Heekeren, Freie Universität Berlin, Germany \\ Reviewed by: \\ Hauke R. Heekeren, Freie Universität Berlin, Germany
}

In 1593, Neapolitan polymath Giambattista della Porta publicly lamented that he was unable to improve his impressive productivity (he had published in areas as diverse as cryptography, hydraulics, pharmacology, optics, and classic fiction). Della Porta was trying to read two books simultaneously by placing both volumes side-by-side, and using each eye independently. To his great surprise, his setup allowed him to only read one book at a time. This discovery arguably marks the first written account of binocular rivalry (Wade, 2000) - a perceptual phenomenon that more than 400 years later still both serves to intrigue as well as to illuminate the limits of scientific knowledge. At first glance, binocular rivalry is an oddball. In every day vision, our eyes receive largely matching views of the world. The brain combines the two images into a cohesive scene, and concurrently, perception is stable. However, when showing two very different images (such as two different books) to each eye, the brain resolves the conflict by adopting a "diplomatic" strategy. Rather than mixing the views of the two eyes into an insensible visual percept, observers perceive a dynamically changing series of perceptual snapshots, with one eye's view dominating for a few seconds before being replaced by its rival from the other eye. With prolonged viewing of a rivalrous stimulus, one inevitably experiences a sequence of subjective perceptual reversals, separated by random time intervals, and this process continues for as long as the sensory conflict is present.

This Frontiers Research Topic focuses on contemporary research on binocular rivalry and related visually multistable phenomena, covering a large variety of topics and techniques. It contains several reviews by leading experts in the field that provide perspectives on important insights that were gained during the past decades of research on rivalry, as well as a focus on outstanding conceptual, methodological, and empirical questions. Additionally, this collection includes research articles using psychophysical, computational, developmental, and imaging techniques that address fundamental questions related to the nature, origins, and neural implications of binocular rivalry. A short overview of the work is outlined in the following paragraphs (please refer to the original articles for further details).
Introducing a novel computational model based on a non-linear algorithm, Lehky (2011) suggests that - at least theoretically - each eye's view can be extracted following binocular integration at later processing stages of the visual system, which could explain some apparent conflicts between previous psychophysical and neurophysiological results. An alternative model that employs an attractor-based neural network architecture previously used to understand working memory, attention, and decision making is presented by Theodoni et al. (2011). One of the hallmarks of binocular rivalry is its unpredictable switching between each eye's views. Kang and Blake (2011) review our current understanding of these dynamic processes, and provide a new framework that integrates the empirical data. Single neuron recordings show perceptual modulation to binocular rivalry as early as V1 (Leopold and Logothetis, 1996; Keliris et al., 2010), ranging all the way to the frontal lobe (Panagiotaropoulos et al., 2012). The long-ranging effects of the neuronal processes that give rise to binocular rivalry can also be measured on the scalp electroencephalogram (EEG). Recent developments in this field, using a combination of new experimental and analytical approaches are reviewed by both Pitts and Britz (2011) as well as Kornmeier and Bach (2012), linking binocular rivalry to other multistable visual phenomena such as the famous "Necker Cube." Wolf and Hochstein (2011) present evidence that binocular rivalry alternations can be modulated by high-level, semantic influences that might originate beyond the visual system. Paffen and Alais (2011) add to the discussion of high-level influences during binocular rivalry by reviewing the most recent literature on attentional influences on perceptual alternations, concluding that high-level selection processes can influence, but are not required to explain the temporal dynamics of binocular rivalry. Dieter and Tadin (2011) provide a complementary review of the interaction between selective attention and binocular rivalry, and place the results in a unifying framework that is based on the classic biased competition model (Desimone and Duncan, 1995). Focusing on low-level influences, on the other hand, Roumani and Moutoussis (2012) review literature on the role of visual adaptation for binocular rivalry alternations. While binocular rivalry is typically studied using artificial stimuli under laboratory conditions, 
Arnold (2011a,b) and O'Shea (2011) engage in a debate on how often binocular rivalry occurs under natural viewing conditions that ends in mutual agreement about the special nature of perceptual alternations. Alais and Parker (2012), on the other hand, demonstrate that even spatially matched images in each eye can rival for perceptual dominance, as long as their temporal modulation is sufficiently different. Andrews and Holmes (2011) revisit the question whether binocular rivalry is mutually exclusive with visual fusion between the two eyes, and present evidence that it is possible to extract stereoscopic depth from stimuli that are perceptually suppressed. Hudak et al. (2011) examine developmental differences of binocular rivalry by measuring its perceptual dynamics in pre-adolescent children. Fahle et al. (2011) combine tactile perceptual responses of subjects with measurements of pupil dilation to demonstrate that rather than constituting an all-or-none event; the internal decision about a perceptual transition seems to build slowly over time. Stuit et al. (2011) present data that suggests additive Gestalt-like grouping effects between binocular rivalry stimuli within and between hemispheres. In the same vein of testing visual interactions between binocular rivalry and other visual phenomena, Masuda et al. (2011) demonstrate that binocular rivalry suppression of key stimulus parts diminishes other visual illusions such as the Craik-O'Brien-Cornsweet effect. Rather than focusing on what is perceptually dominant during binocular rivalry, Zadbood et al. (2011) study what it takes to notice the physical removal of a stimulus that is perceptually suppressed. They find that the result depends on the specific visual feature under scrutiny (Zadbood et al., 2011), suggesting that two equivalent perceptual states can be accompanied by distinct neural events (see also Maier et al., 2007). Denison et al. (2011) tests the influence of perceptual history on

\section{REFERENCES}

Alais, D., and Parker, A. (2012). Binocular rivalry produced by temporal frequency differences. Front. Hum. Neurosci. 6:227. doi: 10.3389/ fnhum.2012.00227

Andrews, T. J., and Holmes, D. (2011). Stereoscopic depth perception during binocular rivalry. Front. Hum. Neurosci. 5:99. doi: 10.3389/ fnhum.2011.00099

Arnold, D. H. (2011a). I agree: binocular rivalry stimuli are common but rivalry is not. Front. Hum. Neurosci. 5:157.doi: 10.3389/fnhum.2011.00157

Arnold, D. H. (2011b). Why is binocular rivalry uncommon? Discrepant monocular images in the real world. Front. Hum. Neurosci. 5:116. doi: 10.3389/fnhum.2011.00116

Denison, R. N., Piazza, E. A., and Silver, M.A. (2011). Predictive context influences perceptual selection during binocular rivalry. Front. Hum. Neurosci. 5:166. doi: 10.3389/fnhum.2011.00166

Desimone, R., and Duncan, J. (1995). Neural mechanisms of selective visual attention. Annu. Rev. Neurosci. 18, 193-222.
Dieter, K. C., and Tadin, D. (2011). Understanding attentional modulation of binocular rivalry: a framework based on biased competition. Front. Hum. Neurosci. 5:155. doi: 10.3389/ fnhum.2011.00155

Fahle, M. W., Stemmler, T., and Spang, K. M. (2011). How much of the "unconscious" is just pre - threshold? Front. Hum. Neurosci. 5:120. doi: 10.3389/ fnhum.2011.00120

Genç, E., Bergmann, J., Tong, F., Blake, R., Singer, W., and Kohler, A. (2011). Callosal connections of primary visual cortex predict the spatial spreading of binocular rivalry across the visual hemifields. Front. Hum. Neurosci. 5:161. doi: 10.3389/ fnhum.2011.00161

Hudak, M., Gervan, P., Friedrich, B., Pastukhov, A., Braun, J., and Kovacs, I. (2011). Increased readiness for adaptation and faster alternation rates under binocular rivalry in children. Front. Hum. Neurosci. 5:128. doi: 10.3389/ fnhum.2011.00128

Kang, M.-S., and Blake, R. (2011). An integrated framework of spatiotemporal dynamics of binocular rivalry. Front.

binocular rivalry, and find that the visual system favors patterns for perceptual dominance if they are predicted by prior stimulation. Pelekanos et al. (2011) also find an effect of stimulus history that they liken to high-level modulation of perceptual selection. Stanley et al. (2011) review data on the initial ("onset") stage of binocular rivalry that follows immediately after the presentation of binocular conflict. Their overview demonstrates that the initiation of binocular rivalry exhibits a variety of idiosyncratic properties that are absent during the ongoing perceptual fluctuations that follow (Stanley et al., 2011). Stienen and De Gelder (2011) present data that suggests that social cues such as fear expression can influence perceptual dominance during rivalry. Stein et al. (2011) investigate in the time it takes for visual stimuli to reach perceptual dominance under continuous flash suppression (Tsuchiya and Koch, 2005), a class of phenomena related to binocular rivalry. They caution against potential over-interpretation of the resulting data for inferences on the brain's processing of unconscious stimuli (Stein et al., 2011). Genç et al. (2011) use diffusion tensor imaging (DTI) to demonstrate a direct relationship between anatomy of transcallosal connections and the perceptual dynamics of rivalry transitions. A refreshing new perspective gets provided by Miller et al. (2011), who suggest that some behavioral characteristics of the fruit fly resemble rivalry-like state changes in humans.

The reviews and empirical articles collected in this research topic demonstrate the many methodological and conceptual advances that have been made by the ever-growing field. The profound insights presented here do not only reflect on our understanding of binocular rivalry and its implications for visual awareness, perceptual organization, and binocular vision, but also have profound implications for our understanding of visual function in general.

Hum. Neurosci. 5:88. doi: 10.3389/ fnhum.2011.00088

Keliris, G.A., Logothetis, N. K., and Tolias, A. S. (2010). The role of the primary visual cortex in perceptual suppression of salient visual stimuli. J. Neurosci. 30, 12353-12365.

Kornmeier, J., and Bach, M. (2012). Ambiguous figures - what happens in the brain when perception changes but not the stimulus. Front. Hum. Neurosci. 6:51. doi: 10.3389/ fnhum.2012.00051

Lehky, S. R. (2011). Unmixing binocular signals. Front. Hum. Neurosci. 5:78. doi: 10.3389/fnhum.2011.00078

Leopold, D. A., and Logothetis, N. K. (1996). Activity changes in early visual cortex reflect monkeys' percepts during binocular rivalry. Nature 379, 549-553.

Maier, A., Logothetis, N. K., and Leopold, D. A. (2007). Context-dependent perceptual modulation of single neurons in primate visual cortex. Proc. Natl. Acad. Sci. U.S.A. 104, 5620-5625.

Masuda, A., Watanabe, J., Terao, M., Watanabe, M., Yagi, A., and Maruya, K. (2011). Awareness of central lumi- nance edge is crucial for the CraikO'Brien-Cornsweet effect. Front. Hum. Neurosci. 5:125. doi: 10.3389/ fnhum.2011.00125

Miller, S. M., Ngo, T. T., and van Swinderen, B. (2011). Attentional switching in humans and flies: rivalry in large and miniature brains. Front. Hum. Neurosci. 5:188. doi: 10.3389/ fnhum.2011.00188

O'Shea, R. P. (2011). Binocular rivalry stimuli are common but rivalry is not. Front. Hum. Neurosci. 5:148. doi: 10.3389/fnhum.2011.00148

Paffen, C. L. E., and Alais, D. (2011). Attentional modulation of binocular rivalry. Front. Hum. Neurosci. 5:105. doi: 10.3389/fnhum.2011.00105

Panagiotaropoulos, T. I., Deco, G., Kapoor, V., and Logothetis, N. K. (2012). Neuronal discharges and gamma oscillations explicitly reflect visual consciousness in the lateral prefrontal cortex. Neuron 74, 924-935.

Pelekanos, V., Roumani, D., and Moutoussis, K. (2011). The effects of categorical and linguistic adaptation on binocular rivalry initial domi- 
nance. Front. Hum. Neurosci. 5:187. doi: 10.3389/fnhum.2011.00187

Pitts, M. A., and Britz, J. (2011). Insights from intermittent binocular rivalry and EEG. Front. Hum. Neurosci. 5:107. doi: 10.3389/fnhum.2011.00107

Roumani, D., and Moutoussis, K. (2012). Binocular rivalry alternations and their relation to visual adaptation. Front. Hum. Neurosci. 6:35. doi: 10.3389/fnhum.2012.00035

Stanley, J., Forte, J. D., Cavanagh, P., and Carter, O. (2011). Onset rivalry: the initial dominance phase is independent of ongoing perceptual alternations. Front. Hum. Neurosci. 5:140. doi: 10.3389/fnhum.2011.00140

Stein, T., Hebart, M. N., and Sterzer, P. (2011). Breaking continuous flash suppression: a new measure of unconscious processing during interocular suppression? Front. Hum. Neurosci. 5:167. doi: 10.3389/ fnhum.2011.00167

Stienen, B.M.C., and De Gelder, B. (2011). Fear modulates visual awareness similarly for facial and bodily expressions. Front. Hum. Neurosci. 5:132. doi: 10.3389/fnhum.2011.00132

Stuit, S. M., Paffen, C. L. E., van der Smagt, M. J., and Verstraten, F. A. J. (2011). What is grouping during binocular rivalry? Front. Hum. Neurosci. 5:117. doi: 10.3389/ fnhum.2011.00117

Theodoni, P., Panagiotaropoulos, T. I., Kapoor, V., Logothetis, N. K., and Deco, G. (2011). Cortical microcircuit dynamics mediating binocular rivalry: the role of adaptation in inhibition. Front. Hum. Neurosci. 5:145. doi: 10.3389/fnhum.2011.00145

Tsuchiya, N., and Koch, C. (2005). Continuous flash suppression reduces negative afterimages. Nat. Neurosci. 8, 1096-1101.

Wade, N. J. (2000). A Natural History of Vision. Boston: MIT Press.

Wolf, M., and Hochstein, S. (2011). Highlevel binocular rivalry effects. Front. Hum. Neurosci. 5:129. doi: 10.3389/ fnhum.2011.00129

Zadbood, A., Lee, S.-H., and Blake, R. (2011). Stimulus fractionation by interocular suppression. Front. Hum. Neurosci. 5:135. doi: 10.3389/ fnhum.2011.00135
Received: 06 August 2012; accepted: 06 September 2012; published online: 25 September 2012.

Citation: Maier A, Panagiotaropoulos TI, Tsuchiya $N$ and Keliris GA (2012) Introduction to research topic-binocular rivalry: a gateway to studying consciousness. Front. Hum. Neurosci. 6:263. doi: 10.3389/ fnhum.2012.00263

Copyright (c) 2012 Maier, Panagiotaropoulos, Tsuchiya and Keliris. This is an open-access article distributed under the terms of the Creative Commons Attribution License, which permits use, distribution and reproduction in other forums, provided the original authors and source are credited and subject to any copyright notices concerning any third-party graphics etc. 